\title{
Ein ethischer Rahmen für den Austausch von Gesundheitsdaten
}

\section{Effy Vayena}

Professorin für Bioethik, Departement für Gesundheitswissenschaften und Technologie, ETH Zürich

Die rasanten Fortschritte an der Schnittstelle der modernen Biomedizin und der Informatik bringen zurzeit eine enorme Menge an gesundheitsbezogenen Daten mit sich. Angesichts des Umfangs und der Komplexität dieser Daten sowie der immer besseren Möglichkeiten, diese zu analysieren, werden sie mitunter auch schon als «Big Data" oder genauer als "Biomedizinische Big Data» bezeichnet. Damit verbunden ist die Erwartung riesiger medizinischer Fortschritte: Die Daten sollen nämlich dazu beitragen, dass Menschen ihren Gesundheitsstatus besser verstehen und auch besser mit Krankheiten umgehen können; gesundheitliche Probleme sollen früher erkannt und die Behandlungen auf die Besonderheiten des einzelnen Patienten angepasst werden. Darüber hinaus könnten Behandlungsergebnisse verbessert, Kosten und Ineffizienz verrin-

\section{Gesundheitsdaten sind eine unschätzbare Ressource, die das Wissen um Krankheiten vergrössern und zu besserer Gesundheits- versorgung beitragen können.}

gert werden. Auch medizinische Entdeckungen und die Entwicklung von Medikamenten sollen sich damit vorantreiben und beschleunigen lassen.

Diesem existierenden und enormen Potenzial der Daten steht jedoch die Tatsache gegenüber, dass die Daten nach wie vor isoliert gespeichert werden, was ihre umfassende Analyse behindert und letztlich den wissenschaftlichen Fortschritt ausbremst. Für diese Situation sind mehrere Gründe verantwortlich, von technischen und praktischen bis hin zu regulatorischen und ethischen. Das Swiss Personalized Health Network (SPHN) ist eine landesweite Initiative [1], um die erforderliche Infrastruktur für den Datenaustausch aufzubauen und damit verbunden für eine bessere Verwendung gesundheitsbezogener Daten in der Forschung.

Datengetriebene Forschung in der Biomedizin und im Gesundheitsbereich insgesamt ist von der Entwicklung geeigneter technischer Standards abhängig, die dafür sorgen, dass Daten sicher ausgetauscht und effizient verarbeitet werden können. Das Hauptziel des SPHN ist daher die Entwicklung von einheitlichen Nor- men und Standards, die den Schweizer Forschenden den Zugriff auf Gesundheitsdaten erleichtern. Diese sind nicht nur für die Schweiz, sondern auch auf globaler Ebene dringend erforderlich. Die Vision des SPHN ist es, dieses Ziel unter Einhaltung hoher ethischer Standards zu erreichen.

H+-Kongress 2017: Mensch und Technologie - digitale Dynamik ohne Grenzen? Technologie und Digitalisierung entwickeln sich mit rasanter Geschwindigkeit. Der H+-Kongress am 8. November 2017 soll dazu beitragen, den Teilnehmenden eine Übersicht zu verschaffen, Fragen zu beantworten sowie Risiken und Chancen aufzuzeigen. Mit dem Kongressprogramm wird das Ziel verfolgt, die wichtigsten Handlungsfelder für die Spitäler und Kliniken auszumachen und eine Brücke zwischen den Akteuren zu schlagen. Unter dieser Prämisse wird die Interaktion am Kongress mit den neuen Knowledge Lounges speziell gefördert. Statt Referate und Workshop-Aufgaben erwarten dort Expertinnen und Experten der Gastgeberinstitutionen dieTeilnehmenden mit Erlebbarem zu unterschiedlichen Aspekten des Kongressthemas.

- Ecole polytechnique fédérale de Lausanne (EPFL): Data-Driven Intelligence

- ETH Zürich: Smart Technologies

- Kommission fürTechnologie und Innovation (KTI): Innovationsförderung im Life-Sciences-Bereich SAMW, SPHN und Science et Cité: Genial digital auch ethisch und sozial?

H+ Bildung und Espace Compétences: Digitalisierung bedeutet Unternehmensentwicklung HINT AG und DIE POST: digitaleTrends - zukunftsweisend und sicher

Der Kongress wird vom SIWF mit 5 Credits anerkannt.

www.hplus-kongress.ch 


\section{Vier Grundprinzipien für den Umgang mit sensiblen Daten}

Angesichts der sensiblen Natur personenbezogener Daten und gesundheitsbezogener Informationen, die aus ihnen gewonnen werden können, besteht Einigkeit darüber, dass sie mit besonderer Sorgfalt zu behandeln sind. Von der Einwilligung zur ursprünglichen Erhebung der Daten über ihre weitere Verwendung bis hin zum Nutzen, der aus dieser Verwendung entsteht,

\section{Das SPHN bietet die Chance, die Forschungs- kultur rund um Gesundheitsdaten grundlegend zu verbessern.}

muss sich die Forschung ernsthaft den ethischen Fragen stellen und diese beantworten. Dazu umfasst das SPHN seit seiner Gründung eine Beratergruppe für ethische und gesellschaftliche Fragen, die sich diesen Aspekten widmet, die Ethical, Legal and Social Implications Advisory Group, kurz ELSIag. Das erste Mandat der ELSlag war die Entwicklung eines ethischen Rahmenwerks (Ethical Framework) für die verantwortungsvolle Datenverarbeitung.

Bei der Aufstellung dieses Dokuments, das auf der SPHN-Website veröffentlicht ist, berücksichtigte die ELSlag nationale und internationale Grundsätze und Richtlinien und führte sowohl innerhalb der eigenen Gruppe als auch mit anderen Gremien innerhalb des SPHN intensive Gespräche. Die ELSlag identifizierte schliesslich vier Grundprinzipien, die das normative Rückgrat jeder verantwortungsvollen Datenverarbei-

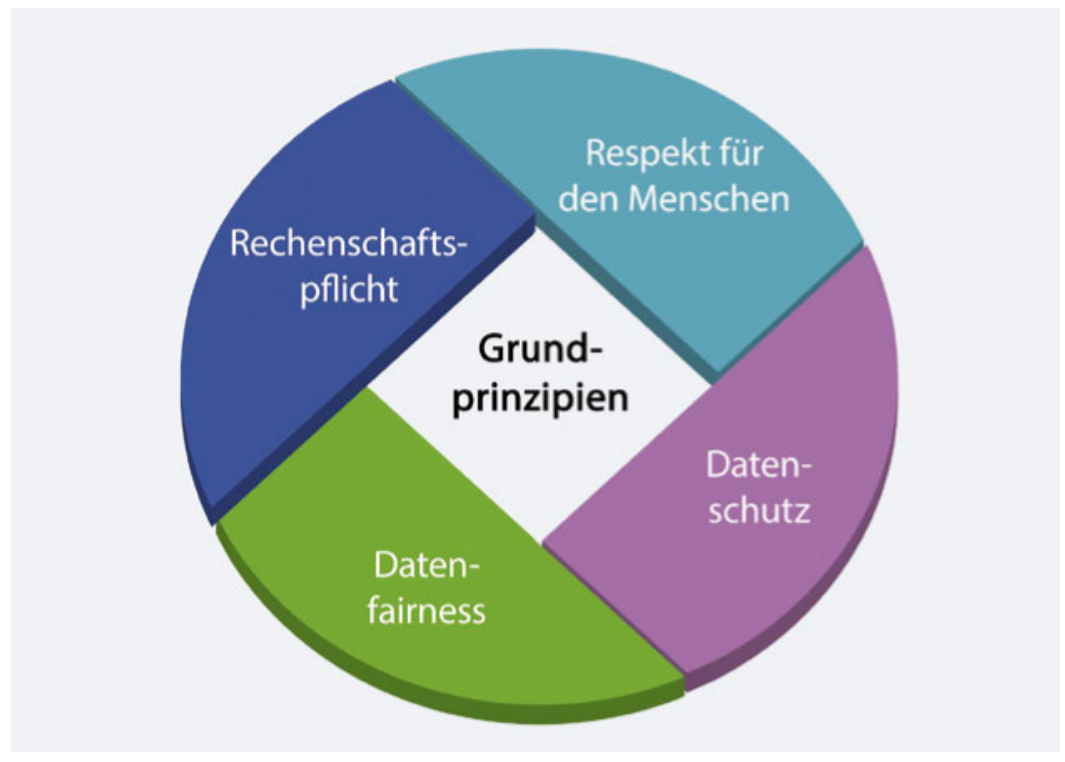

SPHNs vier Grundprinzipien für den verantwortungsvollen Datenaustausch. tung bilden: Respekt für den Menschen, Datenschutz, Datenfairness und Rechenschaftspflicht.

\section{Respekt für den Menschen}

Dieses Prinzip verlangt, dass die Rechte und die Würde von Personen, die Gesundheitsdaten bei Forschungsprojekten oder im Rahmen einer medizinischen Behandlung zur Verfügung stellen, geachtet, geschützt und gefördert werden.

Eine zentrale Anforderung, die sich aus diesem Prinzip ergibt, ist das für die jeweilige Datenverwendung nötige Einverständnis der betroffenen Person. Dazu gehören auch entsprechende Pflichten der Datenverwender, wenn dieses Einverständnis widerrufen wird - ein in der Welt von "Big Data» und ständiger Vernetzung besonders herausforderndes Thema. Darüber hinaus ist dieses Prinzip sowohl für die Mitteilung klinisch umzusetzender Erkenntnisse an Einzelpersonen als auch für die Weitergabe klinischer Forschungsergebnisse an Studienteilnehmende relevant.

\section{Datenschutz}

Dieses Prinzip verlangt den Schutz der Privatsphäre und die Vertraulichkeit von Gesundheitsdaten.

Datenschutz ist ein Grundrecht, dem besondere Aufmerksamkeit zu widmen ist. Umsetzen lässt er sich mittels einer Reihe technischer und verwaltungstechnischer Massnahmen. Zusätzliche Richtlinien innerhalb des ethischen Rahmenwerks spiegeln die Wichtigkeit von Datensicherheit und von Anonymisierungsstandards wider und verlangen laufende Kontrollen bei der Datenverwendung.

\section{Datenfairness}

Dieses Prinzip verlangt, dass sowohl Daten, die zu Forschungszwecken verwendet werden dürfen, als auch Forschungsergebnisse für die weitere Forschung zur Verfügung gestellt werden, um die Wissenschaft als Ganzes voranzubringen.

Gesundheitsdaten sind eine unschätzbare Ressource, die das Wissen um Krankheiten vergrössern und zu besserer Gesundheitsversorgung beitragen können. Aus diesem Potenzial ergibt sich die Verpflichtung, die betreffenden Daten für die ethisch einwandfreie Forschung verfügbar zu machen. Datenfairness fordert den zeitnahen Zugang zu den Daten; es dürfen keine exklusiven Vereinbarungen bestehen, die Forschende am Zugang hindern würden. Die Daten sollten angesichts dieses Prinzips und speziell für die Zwecke des SPHN ohne finanzielle Interessen zugänglich gemacht werden. 


\section{Rechenschaftspflicht}

Dieses Prinzip verlangt, dass Daten fair, transparent und im Rahmen der Gesetze verarbeitet werden.

Alle Mitarbeitenden, die personenbezogene Daten zu Forschungszwecken verarbeiten, müssen für die Folgen zur Verantwortung gezogen werden können, die ihre Handlungen sowohl für die Forschungsteilnehmenden als auch für die Gesellschaft als Ganzes haben können. Die Vorgehensweisen und Mechanismen, die den Umgang mit Daten bestimmen, müssen kontrolliert werden können. Insbesondere haben Forschungsteilnehmer das Recht zu erfahren, wie eine Organisation ihre Daten verarbeitet. Dazu gehören auch die Bedingungen, unter denen sie anderen Benutzern Zugriff auf die Daten gestattet. Transparenz ist ein wesentlicher Bestandteil der Rechenschaftspflicht.

\section{Bedeutung für die Praxis}

Der ethische Rahmen mit diesen vier Grundprinzipien ist so zu verstehen, dass alle Anforderungen gleichzeitig erfüllt werden sollen. Nur durch das Zusammenspiel und nicht durch die Einhaltung einzelner, isolierter Vorgaben sind optimale Ergebnisse sichergestellt. Spezifische, aus den Grundprinzipien abgeleitete Richtlinien sollen ihre synergetische Umsetzung erleichtern. Darüber hinaus ist dieser Rahmen nicht starr, sondern wird vom Austausch mit allen Stakeholdern profitieren und im Zuge wachsender Anforderungen der Netzwerke um weitere Elemente ergänzt.

In den Spitälern werden grosse Mengen von Gesundheitsdaten gesammelt und verwendet. Als wichtige Stakeholder im Gesundheitsbereich geniessen sie das Vertrauen ihrer Patienten und tragen dazu bei, die verantwortungsvolle Verwendung von Gesundheitsdaten aufrechtzuerhalten und weiter aufzubauen. Das ethische Rahmenwerk kann als gemeinsame Basis für die Entwicklung von Governance-Regeln dienen und die Rolle der Spitäler als Verwalter von Gesundheitsdaten stärken. Es soll insbesondere sicherstellen, dass die
SPHN-Initiative angemessenen Standards hinsichtlich ethischer Nachhaltigkeit entspricht, dass sie die Rechte, die Interessen und das Wohlergehen der Forschungsteilnehmer fördert, dem effizienten Ausbau wertvollen Wissens dient und öffentliches Vertrauen rund um die Tätigkeit des Netzwerks schafft. Das Rahmenwerk ist jedoch keine Vorschrift, sondern eher ein gemeinsamer Kodex, der unserem gemeinsamen Interesse als Gesellschaft dient.

Das SPHN bietet die Chance, die Forschungskultur rund um Gesundheitsdaten grundlegend zu verbessern. Dies ist ein Ziel, das viele andere Länder mit uns teilen - die Schweiz ist jedoch in einer einzigartigen Position, um hier voranzugehen. Neben der traditionell hochwertigen biomedizinischen und bioinformatischen Forschung gibt es hier zahlreiche weitere konvergierende Trends, die die Entwicklung der Gesundheitsdatenforschung beflügeln könnten: die Einführung des elektronischen Patientendossiers, die Entwicklung eines allgemeinen Einwilligungsformulars für die Weiterverwendung von Daten (Vorlage Generalkonsent [2]) sowie die Vielzahl der Förderungsmöglichkeiten für Forschungsprojekte auf dem Gebiet der Digitalisierung und verwandter Technologien. Dies alles sind wichtige Indikatoren für ein starkes nationales Interesse und grosses Engagement in diesen Bereichen. Wenn wir uns auf der Basis solider ethischer Grundlagen von diesen Entwicklungen motivieren lassen, kann dies die Art, wie wir forschen, nachhaltig verändern und langfristig auch die Gesundheit des Einzelnen wie auch der Gesamtbevölkerung verbessern.

\section{Literatur}

1 Siehe auch: Lawrence A, Selter L. Swiss Personalized Health Network (SPHN): Die nationale Initiative im Überblick. Schweiz Ärztezeitung 2017;98(19):595-6.

2 Vgl. Vorlage GK 1/2017 der Schweizerischen Akademie der Medizinischen Wissenschaften (SAMW) und der Schweizer Ethikkommissionen für die Forschung am Menschen (swissethics), abrufbar unter: samw.ch/generalkonsent

Bildnachweis

() Effy Vayena / showeet.com 